\title{
Brain Derived Neurotrophic Factor is Decreased in Chronic Fatigue Syndrome and Multiple Sclerosis
}

\author{
Matthew Sorenson ${ }^{1 *}$, Leonard Jason ${ }^{2}$, Jonna Peterson ${ }^{3}$, Joshua Herrington $^{4}$, and Herbert Mathews ${ }^{5}$ \\ ${ }^{1}$ School of Nursing, De Paul University, Chicago, USA \\ ${ }^{2}$ Department of Psychology, De Paul University, Chicago, USA \\ ${ }^{3}$ Rush University, Chicago, USA \\ ${ }^{4}$ Florida International University, Florida, USA \\ ${ }^{5}$ Loyola University Chicago, Chicago, USA
}

"Corresponding author: Matthew Sorenson, Associate Professor/Associate Director DePaul University School of Nursing, 990 West Fullerton Ave., Suite 3000 , Chicago, IL. 60614, USA Tel: 773-325-1887; Fax: 773-325-7282; E-mail: msorenso@depaul.edu

Received date: Mar 2, 2014, Accepted date: Apr 25, 2014, Published date: Apr 30, 2014

Copyright: (c) 2014 Sorenson M, et al. This is an open-access article distributed under the terms of the Creative Commons Attribution License, which permits unrestricted use, distribution, and reproduction in any medium, provided the original author and source are credited.

\begin{abstract}
Objective: This study examined the levels of a major regulator of neuronal survival, brain derived neurotrophic factor (BDNF) in two populations: individuals with multiple sclerosis and chronic fatigue syndrome. BDNF is a protein involved in the maintenance and maturation of both peripheral and central neurons. In patients with multiple sclerosis, BDNF expression is often decreased and believed to reflect ineffective repair mechanisms. As a preliminary exploration, we examined the production of BDNF on the part of peripheral blood mononuclear cells in three groups: patients with Chronic Fatigue Syndrome (CFS [n=15]), patients with multiple sclerosis ( $n=57)$, and a set of putatively healthy controls $(n=37)$.
\end{abstract}

Methods: Mononuclear cells were extracted from peripheral blood samples and cultured for 48 hours. Production of BDNF was evaluated from phyto-haemagglutinin (PHA) and phorbol-12-myristate-13-acetate (PMA) stimulated and unstimulated cells. BDNF levels were determined using a commercially available enzyme linked immuno absorbent assay (sensitivity: 62.5-4,000 pg/mL).

Results: Both CFS and MS samples displayed nearly identical levels of BDNF, levels that were 25 percent of that displayed by the healthy control sample. For unstimulated cells, the BDNF values were $404.71 \mathrm{pg} / \mathrm{ml}$ for the CFS sample, $573.33 \mathrm{pg} / \mathrm{ml}$ for the MS sample and $1,114.15 \mathrm{pg} / \mathrm{ml}$ for the control sample. For stimulated cells, the BDNF values were $442.55 \mathrm{pg} / \mathrm{ml}$ for the CFS sample, $367.33 \mathrm{pg} / \mathrm{ml}$ for the stimulated MS sample, and $1432.24 \mathrm{pg} / \mathrm{ml}$ for the stimulated control sample.

Conclusion: The decreased production of BDNF on the part of MS patients is consistent with the literature. However, the decreased production in those with CFS was unexpected and a novel finding. This finding could reflect a reduced ability to maintain neuronal structure and function in those with CFS. Future studies are needed to evaluate for neuronal damage in those with CFS.

Keywords: Chronic fatigue syndrome; Multiple sclerosis; BDNF; Nerve growth factors; Neutrophic protein

\section{Introduction}

Chronic Fatigue Syndrome (CFS) affects approximately one million Americans, at a cost of billions of dollars to the economy [1]. Emerging evidence suggests the immune system is involved in the pathogenic process of chronic fatigue syndrome [2-5]. While many studies have attempted to clarify the role of the immune system in CFS, little has been accomplished in terms of developing a consensus opinion as to the precise nature of immunologic dysfunction. Immune abnormalities such as low Natural Killer (NK) cell count and dysfunction, along with an imbalance in type I and type II immune response have been suggested, however a great deal of heterogeneity still exists within the CFS literature [6-8]. A guide to future research may be found in work done with Multiple Sclerosis (MS), a neurologic condition in which fatigue is the most common symptom and one that may share some etiologic similarity with CFS [9].

Multiple Sclerosis is a chronic, degenerative neurologic disorder evolving from progressive destruction of the myelin sheath, resulting in a loss of nerve propagation. The destruction of myelin occurs as the result of an autoimmune process in which immune components attack myelin in the central nervous system [10]. The initiation and perpetuation of this pathogenic process is believed mediated by disruptions in the production of certain immune proteins known as cytokines. Of particular note is that the most common symptom displayed by those with MS, is fatigue [11]; a pronounced sense of fatigue that can have pervasive effects on quality of life and functional ability. MS-related fatigue is the leading contributing factor to disability with independent adverse effects on quality of life [12]. While the prevalence of fatigue and its effects on activities of daily living in the MS population are well documented, the causative mechanisms underlying fatigue remain unclear. 
Several potential mechanisms may underlie MS-related fatigue. States of psychological distress/stress have been found associated with fatigue with evidence pointing to the possibility of shared causative pathways $[13,14]$. Heightened psychological stress may be associated with the expression of pro-inflammatory cytokines that are also correlated with fatigue and disease symptomatology. For example, the expression of messenger RNA for the pro-inflammatory cytokine tumor necrosis factor alpha (TNF- $\alpha$ ) has been found associated with fatigue in individuals with MS [15] as well as with perceived stress. This cytokine working in conjunction with interferon-gamma (IFN- $\gamma)$ is believed to be one of the operative players in the demyelinative process. While psychological variables may contribute to fatigue through mediation of immune function, it is just as possible that increasing disease symptomatology could affect ambulative and functional ability with end effects on fatigue. Although, clinical experiences on the part of the lead author have revealed that while patients with MS report significant fatigue, strength and reflex testing during the neurologic examination often evidences no apparent deficit. There may be pathogenic mechanisms leading to fatigue that are distinct from those mechanisms affecting physical mobility. In turn, the administration of immunomodulatory agents during the treatment course can induce a pronounced sense of fatigue; most likely through modulation of cytokine production. It then appears that fatigue is at the very least, mediated by immunologic function.

Recently, evidence has emerged demonstrating close associations between MS and CFS in terms of potential immunologic mechanisms and symptom clusters [9]. Other findings have demonstrated the presence of small white matter hyper intensities in the frontal lobes of a subset of patients with CFS [16]. The presence of such lesions implies the potential presence of immunologic dysfunction within the central nervous system of those with CFS, perhaps similar to that found in MS. In order to investigate the possibility of shared pathogenic markers, this study examined brain derived neurotrophic factor in those with MS and CFS.

Brain Derived Neurotropic Factor (BDNF) is a versatile protein, classified as a member of the neurotrophin family of growth factors, and is found throughout the central nervous system (CNS). It plays a primary role in the formation of the developing nervous system, and contributes to neural plasticity through adulthood. BDNF protein expression has been associated with neural regeneration throughout the body; indeed it is usually found wherever innervations are present. An early animal study found evidence for widespread immunoactivation of BDNF mRNA transcription in the central nervous system (CNS), with concentrations in the lateral septum, bed nucleus of the stria teminalis, medial preoptic nucleus, olivery pretectal nucleus, lateral paragigan to cellular nucleus and the dorsal horn of the spinal cord in adult rats [17].

BDNF is also produced by cells of the immune system. It is secreted by Th1 and Th2 lymphocytes and macrophages, and aids in the growth of cells that may have become damaged by physical injury or neurotropic pathogens. High levels of circulating cytokines in the blood, as in the case of some patients with MS, are known to affect circulating levels of BNDF [18]. This protein is a major component of the neurotrophic theory of depression [19]. The down-regulation of BDNF has been found to lead to neuronal atrophy within the hippocampus, a finding that aids in explaining stress-induced depression [20].

Recently, researchers have focused their attention on the potential of therapeutic and diagnostic uses of BDNF, sometimes with conflicting results. A study that focused on neural plasticity in adult mouse models associated low levels of BDNF with the development of depression [21]. A more recent cohort study examining the genetic component of BDNF reported findings that did not indicate a major influence in the development of depression [22]. Data from a study examining neural lesions in patients with multiple sclerosis (MS) reported that the concentration of BDNF immuno-positive cells were positively correlated with demyelinating activity at lesion sites in the central nervous system [23]. Also, brain derived neurotrophic factor (BDNF) has been shown to be sensitive to exercise in both animal [24] and human populations [25]. So far, very little research has been conducted to examine the role of BDNF in CFS. A literature review yielded only one study [26] that observed an increase of BDNF after an exercise induced state of fatigue in mice. So far, no single clinical standard or diagnostic tool has become available for this debilitating disease. Considering the potential shared immunologic mechanisms between MS and CFS, the study sought to determine the level of BDNF in these disease states in comparison with controls.

\section{Method}

A total of 109 participants (Mean age $=40.5, \mathrm{SD}=13.9$ ) participated in the study. Three separate subgroups were included; 15 participants who met diagnostic criteria for CFS, 57 participants meeting the McDonald criteria for MS [27], and 37 putatively healthy participants who served as controls. Participants with CFS were recruited from a list of individuals who had previously participated in an epidemiological study on CFS. All participants with CFS were positively identified as meeting criteria for CFS [1] and were diagnosed by a physician. All persons involved in the research study were at least 18 years of age, not pregnant, able to speak English, and physically and willfully able to travel to the research center.

All patients with CFS in this study were screened by a licensed physician. Medical examinations included general neurological and physical assessments, as well as a more in-depth evaluation of medical and neurological history. These measures were intended to assess the symptoms, and medical history, of those with CFS in order to rule out any co-occurring medical conditions. Additional medical information deemed relevant to the study was collected to avoid medical confounds for CFS diagnosis, including exposure to HIV/AIDS, tuberculosis, and other non HIV/AIDS sexually transmitted diseases. Information on prescribed and illicit drug use was also assessed and recorded. A history of CFS symptoms was reported as a final measure. Laboratory tests included a complete blood cell count with differential and platelet count, chemistry screen (which assesses thyroid, renal, and liver functioning), Hepatitis B, Lyme disease screen, erythrocyte sedimentation rate, arthritic profile (which includes rheumatoid factor and an-ti-nuclear antibody), HIV screen and urinalysis. A tuberculin skin test was also performed. The project physician performed a detailed medical examination to detect evidence of diffuse cardiac or pulmonary dysfunction, adenopathy, synovitis, neuropathy, myopathy, or hepatosplenomegaly.

A convenience sample of male and female patients diagnosed with MS was recruited from two outpatient neurology clinics. Subjects treated with natalizumab, mitoxantrone or taking oral contraceptives were excluded as were those receiving other immunomodulatory therapies. All subjects were free of steroidal or other immunosuppressant therapy for at least two months prior to enrollment. Also excluded were subjects with current symptoms of 
cardiovascular, respiratory, or neoplastic disease, pregnancy, or nonMS autoimmune disorders.

A control group $(\mathrm{n}=37)$ that was age and gender range (Mean age 43.5, $\mathrm{SD}=2.56$ ) matched to the MS sample population was recruited as part of a separate investigation. The control sample was comprised of healthy individuals with no history of significant illness or injury. All control subjects were free of current symptoms of cardiovascular, respiratory, neoplastic or other autoimmune disorders. The study was approved by the Institutional Review Boards of Loyola University Medical Center, DePaul University and Edward Hines Jr. VA Hospital.

\section{Peripheral blood collection}

Peripheral blood was drawn into lithium citrate tubes and processed within six to eight hours of sample procurement. Plasma samples were obtained from blood collection tubes which were spun for 20 minutes at $1000 \mathrm{X} \mathrm{g}$, and plasma aliquots were pipetted directly from each tube with Phosphate Buffered Saline used for volume replacement. Afterward heparinized peripheral blood was overlaid onto Ficoll/Hypaque and then centrifuged at $1000 \mathrm{X}$ g for $20 \mathrm{~min}$. Cells at the interface were washed with Hank's Balanced Salt Solution (HBSS) and enumerated with a standard hemacytometer.

PBMC's ( 1 x 106) were cultured in RPMI 1640 plus $10 \%$ fetal bovine serum (supplemented with glutamine, penicillin, streptomycin), with (stimulated) and without (constitutive) a mixture of phyto-haemagglutinin (PHA) and phorbol-12-myristate-13-acetate (PMA) (PMA @ 20 ng/well; PHA @ 0.05\%/well) in 24 well plates for $48 \mathrm{hr}$ at $37^{\circ} \mathrm{C}$. After 48 hours, aliquots of supernatant fluid were collected and stored $\left(-70^{\circ} \mathrm{C}\right)$ until analyzed. BDNF levels were determined using commercially available kits purchased from $R \& D$ Systems (Minneapolis, MN).

\section{Data analysis}

All data were initially screened and analyzed descriptively. Demographic (age, gender) and clinical variables were analyzed to determine possible independent effects of these variables on immune profiles. All data were coded and entered into a spreadsheet workbook created in Microsoft Excel; data were also entered in SPSS Version 18
Data entries were compared between Microsoft Excel and SPSS to ensure correct entry. All data analyses were conducted using SPSS Version 18. The alpha level determined prior to analysis was $\mathrm{p}=0.05$. All data prior to the conduction of the analysis were examined for the presence of outliers. In general, an outlier was considered to be a value that lay outside two standard deviations from the mean. Any such outlying values were excluded from analysis. A mixed ANOVA was performed to examine for differences between cell conditions and subject groups. For all statistical analyses performed; Levine's test for equality of variance was applied prior to conduction of t-tests for independent samples. To compare the production between groups, serial $t$-tests for independent samples were performed with the Bonferroni correction.

\section{Results}

\section{Mixed effect analysis}

Using mixed ANOVA analysis, there was no significant constitutive (unstimulated)/stimulated effect $[\mathrm{F}(1,106)=2.34, \mathrm{p}=.13$, partial $\eta \mathrm{p} 2=$. $02]$. However, there were both significant condition $[F(2,106)=$ 23.04, $\mathrm{p}<.00$, partial $\eta \mathrm{p} 2=.30]$ and interaction $[\mathrm{F}(2,106)=35.7, \mathrm{p}<$. 00 , partial $\eta \mathrm{p} 2=.40]$ effects. For the stimulated condition, the control group was significantly higher than the CFS $[t(106)=4.97, \mathrm{p}<.001]$ and MS [t $(106)=7.76, \mathrm{p}<.001]$ groups; however, there were no significant difference for the CFS and MS groups [ $\mathrm{t}(106=0.40, \mathrm{p}=.69$ ].

\section{Level of Constitutive (Unstimulated) PBMC production of BDNF}

The mean level of BDNF produced by PBMC placed in cell culture for 48 hours (unstimulated) and derived from subjects with CFS was $404.71 \mathrm{pg} / \mathrm{ml}(\mathrm{SD}=313.02)$. The level for subjects with MS was slightly higher with a mean of $573.33 \mathrm{pg} / \mathrm{ml}(\mathrm{SD}=405.81)$. These mean values were 25 percent of the level of BDNF produced by control subjects ( $M$ $=1114.15 \mathrm{pg} / \mathrm{ml}, \mathrm{SD}=808.66)$ (Figure 1). Control group levels were significantly higher than the CFS $[\mathrm{t}(106)=4.08, \mathrm{p}<.001]$ and MS [ $\mathrm{t}$ $(106)=4.5, \mathrm{p}<.001]$ groups; however, there were no significant difference between the CFS and MS groups, $\mathrm{t}(106)=1.02, \mathrm{p}=.31$. 


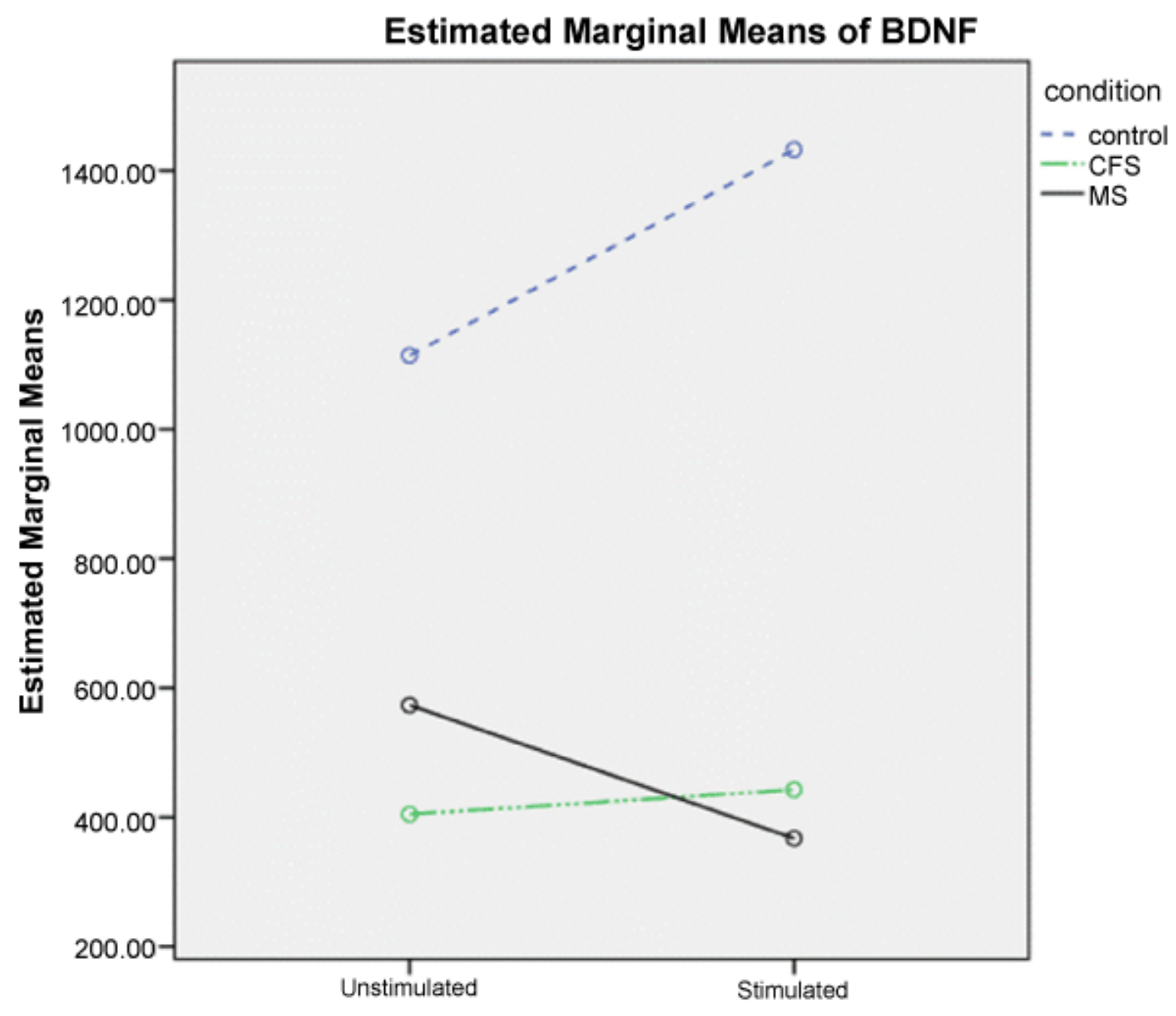

Unstimulated vs. Stimulated

Figure 1. Control group levels

\section{Level of Stimulated PBMC production of BDNF}

The mean level of BDNF produced by PBMC placed in cell culture for 48 hours and stimulated with PMA/PHA for those with CFS was $442.55 \mathrm{pg} / \mathrm{ml}(\mathrm{SD}=323.39)$, which was slightly greater than levels produced constitutively. For the MS sample, the mean level of BDNF was $367.33 \mathrm{pg} / \mathrm{ml}(\mathrm{SD}=241.87)$, which was significantly less than that produced constitutively $[\mathrm{t}(106)=5.29, \mathrm{p}<.001]$. Stimulated PBMC derived from the control group produced a significantly higher mean level of BDNF $(1432.24 \mathrm{pg} / \mathrm{ml}, \mathrm{SD}=1054.99)$ than the unstimulated condition [ $\mathrm{t}(106)=6.58, \mathrm{p}<.001]$. In the CFS sample, there were no significant differences between the stimulated and constitutive conditions $[\mathrm{t}(106)=0.50, \mathrm{p}=.62]$.

\section{Discussion}

These results demonstrate PBMC from patients with CFS or MS produce similar levels of BDNF, and these levels are significantly less than that of healthy control subject PBMC. Lower levels of BDNF have been found in other MS samples [23,28]. However this study is the first to find comparable lower levels among patients with CFS.

In those with MS, fatigue has been found associated with brain lesion. Overall measurements of brain lesion (load) are correlated with fatigue scores with lesions in particular being found in the frontal and parietal regions of the brain [29]. Interestingly, these areas are also found to exhibit lesions in select individuals with CFS [16] and are regions of the brain associated with cognitive function. Not only do patients with MS and CFS share fatigue as a leading symptom, there are often cognitive deficits associated with each condition.

In a population experiencing work associated burnout, serum levels of BDNF were found to be significantly lower than those of a healthy control comparison group. In that study, no differences were found in terms of cortisol levels using a suppression test. It is then possible that low levels of BDNF may be associated with symptoms of altered mood and cognitive function [30]. 
In a murine model of CFS, decreased expression of BDNF in the central nervous system was found associated with hippocampal apoptosis and brain atrophy. These decreases were concomitant with decreases in daily activity and weight loss [31]. An intriguing finding emerged from a study using a murine model of MS (experimental autoimmune encephalomyelitis [EAE]), in which exercise was found to increase the level of BDNF in the central nervous system along with an attenuation of disease severity [32]. It is then apparent that there are ties between the expression of BDNF and exercise. In a human population, decreased levels of BDNF were found at baseline in those with MS as compared to controls. These levels were increased at week four of an exercise intervention, but returned to baseline four weeks later. An acute exercise intervention decreased BDNF levels in both controls and patients with MS during a three hour post-exercise period [33].

The finding of decreased BDNF in individuals with MS is not unexpected $[23,28]$, and may possibly be due to a lack of CD40 markers [23]. The decrease in peripheral BDNF may be reversible through the administration of therapeutic agents in individuals with MS [28,34-36]. The direct administration of cells over-expressing BDNF into the central nervous system of EAE mice has been shown to lead to a less severe form of EAE [37]. The increased levels of BDNF may then have a neuroprotective effect [38] and prevent cellular apoptosis [39]. In turn, BDNF may mediate the release of other immune proteins in the periphery [40]. Murine work has demonstrated the presence of CNS lesion can result in a decrease in the expression of $\mathrm{BDNF}$, leading to a decrease in reparative mechanisms [41]. A similar finding has been demonstrated in human subjects with MS, finding decreased BDNF secretion within the brain to be associated with an increase in disease duration [42].

Other work has found a relative increase in BDNF concentrations on the part of MS patients as compared with controls and those with other neurologic disease states [43]. It is also possible that the particular isoform of BDNF may vary, with MS patients displaying lower levels of mature BDNF in serum [44].

Considering the increasing body of literature demonstrating disruption of immunologic pathways in individuals with CFS $[2,3,45]$, it is important to investigate other potential disease correlates. In a murine model of CFS, a polyphenolic activator (Resveratrol) has been found to increase hippocampal size and improve daily running [46]. The findings from this study and the present provide evidence for $\mathrm{BDNF}$ in the pathogenic process of CFS.

\section{Limitations}

As an initial pilot study, the CFS sample size was relatively small. It will be beneficial for future studies to examine for the presence of neurotrophins in a larger sample. Also, as this was a pilot study, the focus was on BDNF and other neurotrophins were not evaluated. Examining for the presence of other such proteins may provide additional evidence in relation to the disease process.

\section{Conclusion}

The decreased production of BDNF on the part of MS patients is consistent with the literature. However, the decreased production in those with CFS was unexpected and a novel finding. This finding could reflect a reduced ability to maintain neuronal structure and function in those with CFS. Future studies are needed to evaluate for neuronal damage in those with CFS.

\section{Acknowledgement}

The authors wish to thank George Siegel, MD; Chief, Neurology Service, Edward Hines Jr. VA Hospital for his assistance in recruiting and screening patients with multiple sclerosis.

\section{References}

1. Jason LA, Richman JA, Rademaker AW, Jordan KM, Plioplys AV, et al. (1999) A community-based study of chronic fatigue syndrome. Arch Intern Med 159: 2129-2137.

2. Jason L, Sorenson M, Sebally K, Alkazemi D, Lerch A, et al. (2011) Increased HDAC in association with decreased plasma cortisol in older adults with chronic fatigue syndrome. Brain Behav Immun 25: 1544-1547.

3. Jason LA, Sorenson M, Porter N, Belkairous N (2011) An Etiological Model for Myalgic Encephalomyelitis/Chronic Fatigue Syndrome. Neurosci Med 2: 14-27.

4. Torres-Harding S, Sorenson M, Jason L, Maher K, Fletcher MA, et al. (2008). The associations between basal salivary cortisol and illness symptomatology in chronic fatigue syndrome. J Appl Biobehav Res, 13: 157-180.

5. Torres-Harding S, Sorenson M, Jason LA, Maher K, Fletcher MA (2008) Evidence for T-helper 2 shift and association with illness parameters in chronic fatigue syndrome (CFS). Bull IACFS ME 16: 19-33.

6. Patarca-Montero R, Antoni M, Fletcher MA, Klimas NG (2001). Cytokine and other immunologic markers in chronic fatigue syndrome and their relation to neuropsychological factors. Applied neuropsychology 8: 51-64.

7. Klimas NG, Salvato FR, Morgan R, Fletcher MA (1990) Immunologic abnormalities in chronic fatigue syndrome. J Clin Microbiol 28: 1403-1410.

8. Fletcher MA, Maher KJ, Klimas NG (2002). Natural killer cell function in chronic fatigue syndrome. Clinical and Applied Immunology Reviews, 2: 0020129-139.

9. Morris G, Maes, M (2013). Myalgic encephalomyelitis/chronic fatigue syndrome and encephalomyelitis disseminata/multiple sclerosis show remarkable levels of similarity in phenomenology and neuroimmune characteristics. BMC Med 11: 205.

10. Boccaccio GL, Steinman L (1996) Multiple sclerosis: from a myelin point of view. J Neurosci Res 45: 647-654.

11. Bakshi R (2003) Fatigue associated with multiple sclerosis: diagnosis, impact and management. Mult Scler 9: 219-227.

12. Amato MP, Ponziani G, Rossi F, Liedl CL, Stefanile C, et al. (2001) Quality of life in multiple sclerosis: the impact of depression, fatigue and disability. Mult Scler 7: 340-344.

13. Bakshi R, Shaikh ZA, Miletich RS, Czarnecki D, Dmochowski J, et al. (2000) Fatigue in multiple sclerosis and its relationship to depression and neurologic disability. Mult Scler 6: 181-185.

14. Flachenecker P, Kümpfel T, Kallmann B, Gottschalk M, Grauer O, et al. (2002) Fatigue in multiple sclerosis: a comparison of different rating scales and correlation to clinical parameters. Mult Scler 8: 523-526.

15. Flachenecker P, Bihler I, Weber F, Gottschalk M, Toyka KV, et al. (2004) Cytokine mRNA expression in patients with multiple sclerosis and fatigue. Mult Scler 10: 165-169.

16. Lange G, DeLuca J, Maldjian JA, Lee H, Tiersky LA, et al. (1999) Brain MRI abnormalities exist in a subset of patients with chronic fatigue syndrome. J Neurol Sci 171: 3-7.

17. Yan Q, Rosenfeld RD, Matheson CR, Hawkins N, Lopez OT, et al. (1997) Expression of brain-derived neurotrophic factor protein in the adult rat central nervous system. Neuroscience 78: 431-448.

18. Ziemssen T, Kümpfel T, Klinkert WE, Neuhaus O, Hohlfeld R (2002) Glatiramer acetate-specific T-helper 1- and 2-type cell lines produce BDNF: implications for multiple sclerosis therapy. Brain-derived neurotrophic factor. Brain 125: 2381-2391. 
19. Dawood T, Anderson J, Barton D, Lambert E, Esler M, et al. (2007) Reduced overflow of BDNF from the brain is linked with suicide risk in depressive illness. Mol Psychiatry 12: 981-983.

20. Duman RS, Heninger GR, Nestler EJ (1997) A molecular and cellular theory of depression. Arch Gen Psychiatry 54: 597-606.

21. Kuipers SD, Bramham CR (2006) Brain-derived neurotrophic factor mechanisms and function in adult synaptic plasticity: new insights and implications for therapy. Curr Opin Drug Discov Devel 9: 580-586.

22. Chen L, Lawlor DA, Lewis SJ, Yuan W, Abdollahi MR, et al. (2008). Genetic association study of BDNF in depression: Finding from two cohort studies and a meta-analysis. Am. J. Med. Genet. Part B Neuropsychiatr. Genet. American Journal of Medical Genetics, Part B: Neuropsychiatric Genetics, 147: 814-821.

23. Azoulay D, Urshansky N, Karni A (2008) Low and dysregulated BDNF secretion from immune cells of MS patients is related to reduced neuroprotection. J Neuroimmunol 195: 186-193.

24. Rasmussen P, Brassard P, Adser H, Pedersen MV, Leick L, et al. (2009) Evidence for a release of brain-derived neurotrophic factor from the brain during exercise. Exp Physiol 94: 1062-1069.

25. Gold SM, Schulz KH, Hartmann S, Mladek M, Lang UE, et al. (2003). Basal serum levels and reactivity of nerve growth factor and brainderived neurotrophic factor to standardized acute exercise in multiple sclerosis and controls. J Neuroimmunol 138: 99-105.

26. Chen R, Moriya J, Yamakawa J, Takahashi T, Li Q, et al. (2008) Brain atrophy in a murine model of chronic fatigue syndrome and beneficial effect of Hochu-ekki-to (TJ-41). Neurochem Res 33: 1759-1767.

27. Polman CH, Reingold SC, Edan G, Filippi M, Hartung HP, et al. (2005) Diagnostic criteria for multiple sclerosis: 2005 revisions to the "McDonald Criteria". Ann Neurol 58: 840-846.

28. Azoulay D, Vachapova V, Shihman B, Miler A, Karni A (2005) Lower brain-derived neurotrophic factor in serum of relapsing remitting MS: reversal by glatiramer acetate. J Neuroimmunol 167: 215-218.

29. Sepulcre J, Masdeu JC, Goñi J, Arrondo G, Vélez de Mendizábal N, et al. (2009) Fatigue in multiple sclerosis is associated with the disruption of frontal and parietal pathways. Mult Scler 15: 337-344.

30. Onen Sertoz O, Tolga Binbay I, Koylu E, Noyan A, Yildirim E, et al. (2008) The role of BDNF and HPA axis in the neurobiology of burnout syndrome. Prog Neuropsychopharmacol Biol Psychiatry 32: 1459-1465.

31. Bernardes D, Oliveira-Lima OC, Silva TV, Faraco CC, Leite HR, et al. (2013) Differential brain and spinal cord cytokine and BDNF levels in experimental autoimmune encephalomyelitis are modulated by prior and regular exercise. J Neuroimmunol 264: 24-34.

32. Castellano V, White LJ (2008) Serum brain-derived neurotrophic factor response to aerobic exercise in multiple sclerosis. J Neurol Sci 269: 85-91.

33. Blanco Y, Moral EA, Costa M, Gomez-Choco M, Torres-Peraza JF, et al. (2006). Effect of glatiramer acetate (Copaxone) on the immunophenotypic and cytokine profile and BDNF production in multiple sclerosis: a longitudinal study. Neurosci Lett 406: 270-275.

34. Caggiula M, Batocchi AP, Frisullo G, Angelucci F, Patanella AK, et al. (2006). Neurotrophic factors in relapsing remitting and secondary progressive multiple sclerosis patients during interferon beta therapy. Clin Immunol 118: 77-82.

35. Hamamcioglu K, Reder AT (2007) Interferon-beta regulates cytokines and BDNF: greater effect in relapsing than in progressive multiple sclerosis. Mult Scler 13: 459-470.

36. Linker RA, Lee DH, Demir S, Wiese S, Kruse N, et al. (2010). Functional role of brain-derived neurotrophic factor in neuroprotective autoimmunity: therapeutic implications in a model of multiple sclerosis. Brain 133: 2248-2263.

37. Bayas A, Hummel V, Kallmann BA, Karch C, Toyka KV, et al. (2002) Human cerebral endothelial cells are a potential source for bioactive BDNF. Cytokine 19: 55-58.

38. De Santi L, Cantalupo L, Tassi M, Raspadori D, Cioni C, et al. (2009) Higher expression of BDNF receptor gp145trkB is associated with lower apoptosis intensity in $\mathrm{T}$ cell lines in multiple sclerosis. J Neurol Sci 277: 65-70.

39. Bayas A, Kruse N, Moriabadi NF, Weber F, Hummel V, et al. (2003). Modulation of cytokine mRNA expression by brain-derived neurotrophic factor and nerve growth factor in human immune cells. Neurosci Lett 335: 155-158.

40. VonDran MW, Singh H, Honeywell JZ, Dreyfus CF (2011) Levels of BDNF impact oligodendrocyte lineage cells following a cuprizone lesion. J Neurosci 31: 14182-14190.

41. Weinstock-Guttman B, Zivadinov R, Tamaño-Blanco M, Abdelrahman $\mathrm{N}$, Badgett D, et al. (2007) Immune cell BDNF secretion is associated with white matter volume in multiple sclerosis. J Neuroimmunol 188: 167-174.

42. Gielen A, Khademi M, Muhallab S, Olsson T, Piehl F (2003). Increased brain-derived neurotrophic factor expression in white blood cells of relapsing-remitting multiple sclerosis patients. Scand J Immunol 57: 493-497.

43. Tongiorgi E, Sartori A, Baj G, Bratina A, Di Cola F, et al. (2012) Altered serum content of brain-derived neurotrophic factor isoforms in multiple sclerosis. J Neurol Sci 320: 161-165.

44. Brenu EW, Huth TK, Hardcastle SL, Fuller K, Kaur M, et al. (2014) Role of adaptive and innate immune cells in chronic fatigue syndrome/ myalgic encephalomyelitis. Int Immunol 26: 233-242.

45. Moriya J, Chen R, Yamakawa J, Sasaki K, Ishigaki Y, et al. (2011). Resveratrol improves hippocampal atrophy in chronic fatigue mice by enhancing neurogenesis and inhibiting apoptosis of granular cells. Biol Pharm Bull 34: 354-359.
This article was originally published in a special issue, entitled:

"Neurodegenerative Diseases: Symptoms and Therapeutics", Edited by Dr. Jin J Luo, Temple University School of Medicine, USA 